\title{
Dynamic Tracking Experimental Research of Adjusting System for the Subreflector of 65 Meters Radio Telescope
}

\author{
Yuchao Dou \\ School of Mechanical Engineering. Yanshan University \\ Qinhuangdao, Hebei, P. R. China \\ ycdou@ysu.edu.cn
}

\author{
Yulei Hou \\ School of Mechanical Engineering. Yanshan University \\ Qinhuangdao, Hebei, P. R. China
}

\author{
Hang Dong \\ School of Mechanical Engineering. \\ Yanshan University \\ Qinhuangdao, Hebei, P. R. China
}

\author{
Jiantao Yao \\ School of Mechanical Engineering. \\ Yanshan University \\ Qinhuangdao, Hebei, P. R. China
}

\author{
Yongsheng Zhao \\ School of Mechanical Engineering. \\ Yanshan University \\ Qinhuangdao, Hebei, P. R. China \\ yszhao@ysu.edu.cn
}

\begin{abstract}
In order to improve the dynamic tracking ability of adjusting system for the subreflector of 65 meters radio telescope, and lay the foundation for the calibration works, PID parameters were adjusted through parabolic speed response and ramp position response based on electrical control system. Circle tracking test were carried out using laser tracker, the result demonstrate that the dynamic tracking ability of subreflector control system is improved and calibration can carry out.
\end{abstract}

Keywords-65 meters radio telescope; subreflector; adjusting system; dynamic tracking; PID parameters

\section{INTRODUCTION}

In order to completion of VLBI (Very Long Baseline Interferometry) in the lunar exploration mission and satisfy the demand for deep space exploration in the future, a radio telescope with the main reflector's diameter is 65 meters will be built in Shanghai by the cooperation of Chinese Academy of Science and Shanghai City, which will be the largest in Asia and possess international advanced performance

The subreflector's diameter is 6.5 meters and the weight is 1.6 tons, it is supported by four truss which connected to main reflector, the distance between subreflector and main reflector center is 30 meters for Shanghai 65 meters radio telescope. A Stewart parallel robot as the subreflector adjusting mechanism is used to compensate the subreflector pointing error was caused by truss deformation. In order to realize the design target: positioning error less than $0.05 \mathrm{~mm}$, orientation error less than $10^{\prime \prime}$, adjusting time less than 30 s, usually the structure parameters need calibrated base on stable electrical control system, so it's necessary to adjusting PID parameters to improve the dynamic tracking ability. The paper ${ }^{[1,2]}$ proposed nonlinear PID control and adaptive interacting PID supervision method to control flexible parallel robot, and discussed the position and orientation dynamic tracking ability. Yu et ${ }^{[3]}$ designed a 3-RRR parallel robot experiment system and kinematic simulation was carried out to demonstrate the control strategy is feasible. Xu et ${ }^{[4]}$ proposed predictive control strategy for a parallel structure which used as telescope support structure. $\mathrm{Yu}$ et ${ }^{[5]}$ proposed a new method for calibrating parallel robot, the calibration method with a coordinate measuring machine is verified through random pose test experiment. Wang et ${ }^{[6]}$ deals with the experimental study of a redundantly actuated parallel manipulator for a 5-DOF hybrid machine tool, the machine experiment demonstrate that the machine's applicability. Wang et ${ }^{[7]}$ designed a planar 1R2T wire-driven parallel robot with the aim of realizing rehabilitation training, the trajectory control experiment results show that the motion planning method and the experiment calibration method is suitable for other wire-driven parallel system. Li et ${ }^{[8]}$ performed experiment to investigate indexes of accuracy sensitivity, the regression equations are established to analyze the significance of various factors according to experiment results.

This paper developed the adjusting control system for the subreflector of 65 meters radio telescope, and set the PID parameters by executing parabolic speed response and ramp position response. The performance of dynamic tracking is also analyzed. At last, the circle trace track experimentation was carried out to test the tracking ability of subreflector which lay foundation for the calibration in the next step.

\section{ADJUSTING MECHANISM FOR THE SUBREFLECTOR}

The diagram of subreflector adjusting mechanism is shown in Fig.1, it is composed of the fixed platform, the moving platform and six drive limbs. The drive limb connected to fixed platform and moving platform through universal pairs and sphere pairs, respectively. The prismatic pair in the middle of the drive limb is selected as the actuator. The universal pairs $B_{i}(i=1,2 \ldots 6)$ are divided into three groups uniform distribution in radius of circles $R$, universal pair angle

Chinese Academy of Sciences, Shanghai major cooperation project. 
between each group $\phi_{1}$, within each group angle between two universal pairs $\theta_{1}$. sphere pairs $b_{i}(i=1,2 \ldots 6)$ divide into three groups uniform distribution in radius of circles $r$, sphere pairs angle between each group $\phi_{2}$, within each group angle between two universal pairs $\theta_{2}$.

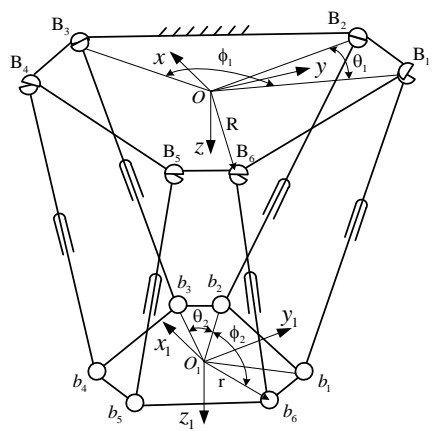

Fig 1. Diagram of subreflector adjusting mechanism

The prototype of subreflector adjusting mechanism is shown in Fig.2, and the structure parameters are $R=2060 \mathrm{~mm}, \phi_{1}=120^{\circ}, \theta_{1}=10^{\circ}, r=1555 \mathrm{~mm}, \phi_{2}=120^{\circ}$, $\theta_{2}=10^{\circ}$. Inverse kinematics is can be obtain according to the structure $^{[9]}$.

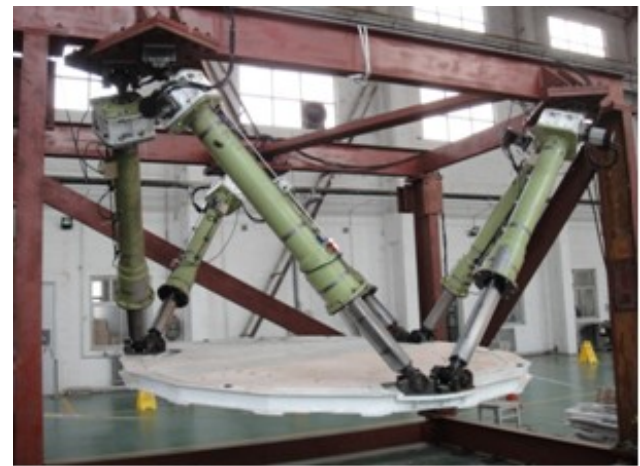

Fig 2. Prototype of the subreflector adjusting mechanism

\section{ELECTRICAL CONTROL SYSTEM OF THE ADJUSTING MECHANISM FOR THE SUBREFLECTOR}

The electrical control system is shown in Fig 3. The industrial personal computer in the charge of receiving the subreflector adjust command from main computer, then calculate and generate different axis movement commands according to inverse kinematics of parallel adjusting mechanism. The controller will control the subreflector move to the expected pointing after received axis moving command from industrial control computer.

In order to eliminate the influence of reverse clearance from ball screw and reducer in linear motion unit, the absolute displacement data from magnetic linear displacement transducer installed in limbs is taken as position loop data. Three grades security are set up to improve the safety in operation process of the equipment. Software limit is taken as the first level security, the controller will control motors decelerate stopping according to specified acceleration when the position loop data beyond the threshold. The second level security sensor is proximity sensor and will become effective if the first level lose effectiveness, the process decelerate stopping as the software limit trigger. If the two levels security both lose effectiveness, the third level security will cut off power supply immediately to ensure the equipment safety by press the emergency stop switch.

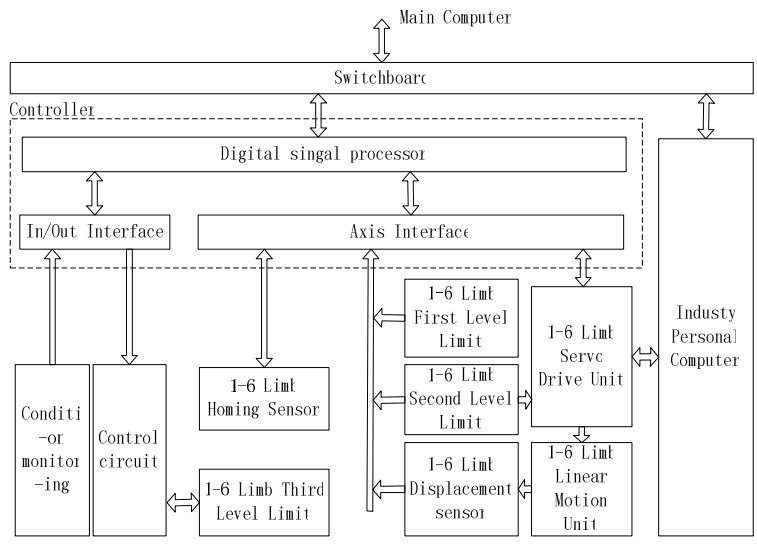

Fig 3. Diagram of electrical control system

Condition monitoring circuit monitor the status of control system and send it to controller, so that operator can grasp equipment status. Homing sensor is installed in a fixed position along the limb, that can rebuild home point by execute homing process when magnetic linear displacement transducer lose effectiveness, which can avoid the problems from second calibration.

\section{DYNAMIC TRACKING EXPERIMENTATION OF ADJUSTING SYSTEM FOR THE SUBREFLECTOR}

The control system interface of subreflector adjusting is shown in Fig.4. In the purpose of improving dynamic tracking performance of adjusting control system and lay foundation for calibration in the next step, speed loop and position loop parameter are adjusted through parabolic response speed and ramp position response.

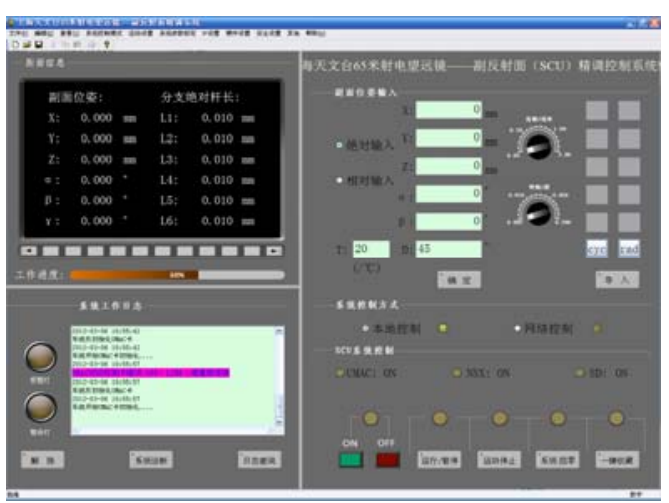

Fig 4. The control system interface of subreflect adjusting system

Fig. 5 is parabolic speed response without PID parameters adjusted. From the Fig. 5, it can be seen that speed response lag 
in commanded velocity serious, the maximum retardation time up to $100 \mathrm{~ms}$ in the time of acceleration reversing. The following error is smooth in the constant acceleration phase, but it turn into fluctuation serious during variable acceleration, the maximum following error is $1.2 \mathrm{~mm} / \mathrm{s}$.

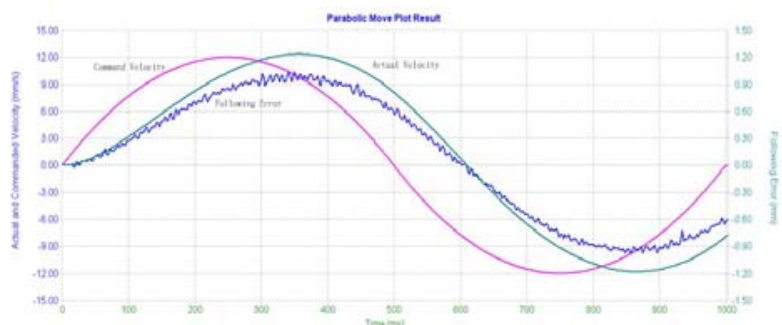

Fig 5. Parabolic speed response of subreflector control system without adjust

Judging by the response it should increase proportion gain and reduce damping, integral gain remain unchanged until overshoot is appeared, adjusting integral gain according to the steady error situation. The results are shown in Fig. 6.

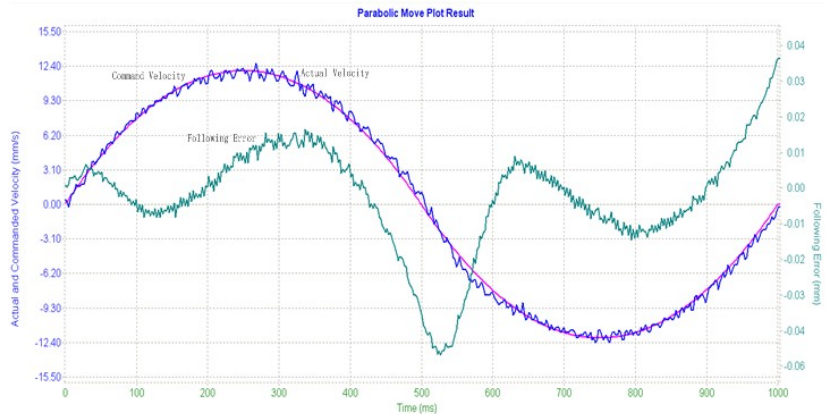

Fig 6 . Parabolic speed response of subreflector control system after adjust

After PID was adjusted, the parabolic response speed curve can be a very good envelope commanded velocity curve, the maximum speed tracking error dropped to $0.05 \mathrm{~mm} / \mathrm{s}$.

As the outside loop of servo control system, the position loop tracking performance directly affect the dynamic tracking precision, the initial state position loop ramp response as shown in Fig. 7.

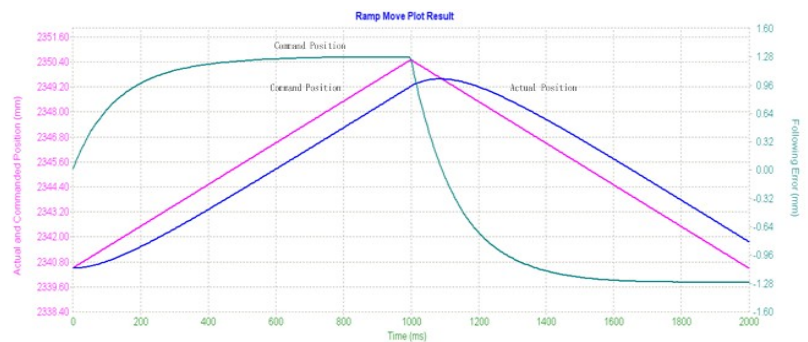

Fig 7. Ramp position response of subreflector control system without adjust

From Fig. 7, it can be known that position ramp response lag in commanded position serious. The maximum following error is $0.64 \mathrm{~mm}$ in starting, the average following error is $1.28 \mathrm{~mm}$ in uniform phase. However, that the following error is a constant although position ramp response is lag in commanded, this show that the system can maintain stable under external disturbances, so we can increase the proportion gain of position loop and appropriately increase damping to reduce tracking error according to the overshoot situation at reversing point. The results are shown in Fig. 8.

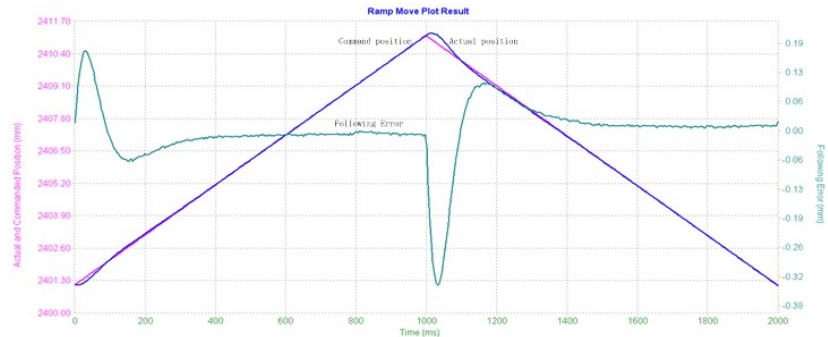

Fig 8. Ramp position response of subreflector control system after adjust

The maximum position following error drops to $0.18 \mathrm{~mm}$ at starting, and the average position following error approaches to $0.01 \mathrm{~mm}$ in constant speed phase.

API T3 laser tracker, shown in Fig.9, was used to tracking circular trajectory $\mathrm{r}=200 \mathrm{~mm}$, and sampling 1101 points to calculate the absolutely following error. The result is shown in Fig. 10.

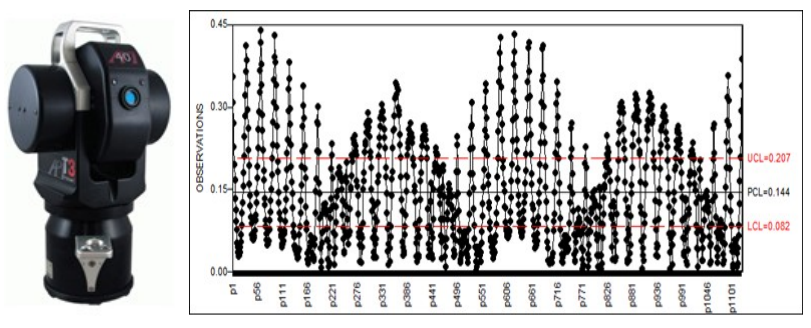

Fig 9. laser tracker

Fig 10. Circular trajectory following error

The maximum following error is $0.439 \mathrm{~mm}$, the minimum following error is $0.0015 \mathrm{~mm}$, the average of following error is $0.144 \mathrm{~mm}$, which lay a foundation for calibration scheme design and calibration effect evaluation.

\section{CONCLUSIONS}

The adjusting control system for the subreflector of the 65 meters radio telescope has been developed in this paper. PID parameters tuning obtained through parabolic velocity response and ramp position response will be helpful to improve the system dynamic tracking performance. Circular trajectory test was carried out to acquire the as far as possible real data of tracking performance. The method of PID parameters tuning and process discussed in this paper should be valuable for the development of other parallel mechanism control systems.

\section{REFERENCES}

[1] J. L. Du, B. Hong, B. Y. Duan. Tracking Control of Cable-driven Parallel Robots Considering Cable Sag Effects [J]. Journal of Mechanical Engineering.2010,3,pp.17-28.

[2] X. C. Duan, Y. Y. Chou, B. Y. Duan etc. Adaptive Interactive PID Supervisory Control of the Macro-micro Parallel Manipulator [J]. Journal Of Mechanical Engineering. 2010,46(1), pp.10-17.

[3] X. T. Zhang, J. X. Yang, Y. Q. Yu. Experiment System for the HighSpeed Flexible Planar 3-RRR Parallel Robot [J]. Machine Design and Research, 2008,24(5), pp.39-46. 
[4] S. G. Xu, H. Wang. A Control Scheme for the Parallel Robot Used a s the Supporting Mechanism of an Astronomical Telescope [J]. Astronomical Research \& Technology,2009, 6(3), pp.228-233.

[5] D. Y. Yu, H. R. Li, W. F. Chen. Kinematic Calibration of Parallel Robots for Docking Mechanism Motion Simulation [J], Int J Adv Robotic Sy, 2011,8(4), pp.158-165.

[6] L. P. Wang, J. Wu, J. S. Wang, Z. You. An Experimental Study of a Redundantly Actuated Parallel Manipulator for a 5-DOF Hybrid Machine Tool [J], Mechatronics,14(1), pp.72-81.
[7] K. Y. Wang, L. X. Zhang, L. Wang, F. Q. Liu. Motion planning and experiment of a planar wire-driven parallel robot [C], Mechanic Automation and Control Engineering, June 2010.pp. 26-28 .

[8] J. G. Li, J. Ding, H. J. Jing, Y. X. Yao. Accuracy Analysis of Stewart Parallel Manipulator Based on Uniform Experiment Design [J], Advanced Materials Research,2012.pp.715-720.

[9] Y. L. Hou, Y. B. Duan, J. T. Yao, etc. Configuration Optimization and Static Analysis of Adjusting Parallel Mechanism for the Sub-reflector of Antenna [J], Advanced Materials Research,Vol.338 (2011) pp.425-430. 